\title{
Qualidade de vida dos servidores em processo de envelhecimento - IFPR Campus Palmas
}

\author{
Quality of life and aging among \\ federal civil servants - IFPR Campus \\ Palmas
}

FisiSenectus . Unochapecó Ano 3, n. 2 - Jul/Dez. 2015 p. $13-23$

Cezar Grontowski Ribeiro. cezar.ribeiro@ifpr.edu.br

Educador Físico. Mestre em Ciências da Saúde pela Universidade Comunitária da Região de Chapecó-SC. Coordenador do curso de Educação Física e do Projeto Melhoria da Saúde e Qualidade de Vida no IFPR Campus Palmas.

Aline Mati Shimosaka. alineshimosaka@hotmail.com Acadêmica do curso de Educação Física do Instituto Federal do Paraná - Campus Palmas. Bolsista de Iniciação Científica do Projeto Melhoria da Saúde e Qualidade de Vida no IFPR Campus Palmas (PIBIC-FA).

David da Costa. david16-@live.com Acadêmico do curso de Educação Física do Instituto Federal do Paraná - Campus Palmas. Bolsista de Iniciação Científica do Projeto Melhoria da Saúde e Qualidade de Vida no IFPR Campus Palmas (PIBIC-FA).

\section{Resumo}

Introdução: todo ano, cerca de 650 mil brasileiros ingressam na chamada terceira idade. Atualmente, percebe-se maior prevalência de doenças crônico-degenerativas, interferindo na qualidade de vida (QV) desses idosos. Objetivos: verificar a QV do servidor federal que se encontra na fase da senescência, atuante em instituição situada no sul do estado do Paraná. Materiais e métodos: participaram do estudo 36 servidores: docentes, técnicos administrativos e terceirizados, de ambos os sexos, com faixa etária entre 40 e 57 anos. Os dados foram obtidos por meio do Whoqol-bref (avaliação da qualidade de vida), IPAQ (avaliação do nível de atividade física) e Questionário da ABEP (avaliação da condição socioeconômica). A pesquisa foi aprovada pelo Comitê de Pesquisa e Extensão. Resultados: observa-se que o nível de condição econômica parece ser fundamental na concepção da QV dos sujeitos, apresentando índices melhores em indivíduos com maior poder de renda. 0 nível de atividade física não se mostrou interferente na percepção da QV para essa população. A proximidade da velhice não parece ser ainda um fator que interfere nas percepções de QV dos sujeitos da pesquisa. Conclusão: A atividade física não apresentou interferência, ao passo que a renda mostrou-se fundamental para índices mais positivos de QV.

\section{Palavras-chave}

Qualidade de vida. Envelhecimento. Trabalhadores. Atividade motora.

\section{Fisǐ̉enectus}




\begin{abstract}
Introduction: every ear about 650 thousand Brazilians join the called third age. Currently there is higher prevalence of cronic deseases, affecting the quality of life (QOL) of the elderly. Objective: to verify the QOL of the federal server that is at the stage of senescence, active in federal institution in the southern state of Paraná. Materials and methods: Study participants were 36 teachers servers, outsourced administrative and technical, of both sexes, aged between 40 and 57 years. Data were obtained through the Whoqol-bref (assessment of quality of life), IPAQ (assessment of the level of physical activity) and the ABEP questionnaire (evaluation of socio-economic status). The study was approved by the Research Ethics Committee. Results: it is observed that the level of economic status appears to be fundamental in the design of QOL of subjects, presenting best rates in individuals with greater power of income. The physical activity level were not interfering in the perception of QOL for this population. The proximity of old age does not seem to be even a factor that interferes with QOL perceptions of the research subjects. Conclusion: Physical activity showed no interference, while the income proved crucial to the most positive indices of QOL.
\end{abstract}

\title{
Keywords
}

Quality of life. Aging. Workers. Motor activity.

\section{Introdução}

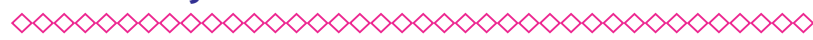

No Brasil, a cada ano, aproximadamente 650 mil pessoas ingressam na classe considerada terceira idade ${ }^{1}$. Mais importante que o número de idosos que se amplia anualmente é a forma como as pessoas chegam nessa fase da vida. Os avanços tecnológicos, a descoberta de novos medicamentos e tecnologias para o diagnóstico de doenças, entre outras características, foram fatores que contribuíram para o aumento da Iongevidade humana. Mas enquanto a mortalidade por males infectocontagiosos foi reduzida, percebe-se um aumento no número de doenças crônico-degenerativas ocasionadas por essa nova tendência².

Os efeitos do envelhecimento sobre o corpo humano são objeto de inúmeros estudos. Envelhecer pode ser caracterizado como processo dinâmico e progressivo, em que ocorrem importantes transformações nos aspectos morfofuncionais, biofisiológicos e psicossociais. Nesse período, as perdas são graduais, ocorrendo em ritmo diferenciado e individual e diversas questões apresentam-se como interferentes (estilo de vida, alimentação, nível de atividade física, entre outros) ${ }^{3}$.

A qualidade de vida (QV) aparece nesse período como fator importante de análise, já que possibilita compreender mecanismos que afetam diretamente na forma como as pessoas veem sua vida e é considerada boa ou excelente quando apresenta minimamente as condições ao sujeito para que ele possa desenvolver suas capacidades e viver plenamente nas variadas dimensões da vida humana-familiar, laboral, social, entre outras ${ }^{4}$.

A QV é fator de constante preocupação tanto nas esferas políticas como da população de maneira geral. Está associada à própria percepção do indivíduo sobre a sua maneira de situar-se no contexto cultural, considerando suas preocupações, objetivos, expectativas, satisfação no emprego, aspectos do estilo de vida e do ambiente em que se vive, e mesmo nas relações sociais ou familiares ${ }^{5}$, além dos aspectos subjetivos inerentes ao ser humano que interferem diretamente na sua mensuração e se apresentam como fundamentais para essa compreensão.

A qualidade de vida no trabalho (QVT) é uma preocupação crescente e que tem sido objeto de pesquisas que procuram conhecer a satisfação e bem-estar do ser humano no ambiente laboral. Como na maioria das profissões, o servidor dedica pelo menos $1 / 3$ da carga horária diária ao seu ambiente de trabalho. Dessa maneira, é preciso adequar os espaços, torná-los seguros e confortáveis, objetivando comodidade e bemestar, que poderão levar a uma maior eficiência e produtividade. Nesse sentido, é necessário agregar 
ações que tenham por intuito a implantação de melhorias que impactem diretamente na QVT dos trabalhadores $^{6}$.

A QV também é percebida como satisfação das necessidades humanas, abrangendo não somente aspectos subjetivos como felicidade, espiritualidade etc., mas também situações objetivas, como salário, condições de trabalho, entre outros, sendo que para haver produtividade é preciso que o sujeito esteja contente com suas funções, responsabilidades e remuneração ${ }^{7}$.

O Brasil, assim como a maioria dos países em desenvolvimento, tem buscado estratégias para melhorar a QV das pessoas. Diante disso, avaliar essa instância nas pessoas adultas pode contribuir de maneira significativa para compreensão dos fatores condicionantes do processo de envelhecer. Na esfera do serviço público, avaliar a QV dos servidores pode incentivar a implantação de políticas e práticas que visem melhorar as condições desses trabalhadores.

0 olhar apresentado leva a crer que em todas as fases do desenvolvimento e, dessa maneira, também na vida adulta e velhice, a satisfação do ser humano com a vida está diretamente ligada ao meio em que o indivíduo insere-se, às aspirações que possui e à disponibilidade de recursos e materiais, ou seja, ao atendimento de suas necessidades.

É importante ressaltar que a QV pode ser afetada por diversos aspectos derivados da própria condição de trabalho, como as relacionadas aos domínios físico, psicológico, social e das relações com o ambiente. Considerando que esses fatores influenciam-se internamente nos sujeitos e em diferentes grupos populacionais, o estudo ora apresentado teve como objetivo geral analisar a qualidade de vida dos servidores em processo de envelhecimento do IFPR Campus Palmas, sendo que os resultados encontrados podem nortear ações para melhoria dos quadros constatados, além de servir como indicadores para outras pesquisas com populações de características semelhantes.

\section{Materiais e métodos}

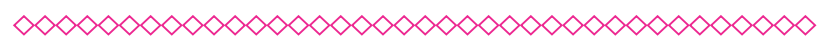

A pesquisa é quantitativa, de cunho descritivo transversal, realizada a partir da seleção intencional dos participantes: 36 servidores docentes, técnicos administrativos e serviços terceirizados lotados no IFPR, com idade entre 40 e 57 anos de idade, de ambos os sexos. A amostra corresponde a $100 \%$ dos servidores em atividade no campus com essas características. Foi utilizado como critério de inclusão ser servidor ativo, com pelo menos um ano de atividade no local e idade entre 40 e 59 anos.

Para coleta de dados foram usados os seguintes instrumentos:

1 - Versão em português do Instrumento de Avaliação de Qualidade de Vida WhoqolBref, conforme protocolo ${ }^{8}$. Na versão breve, 0 questionário é estruturado com 26 questões, sendo que 24 delas são agregadas em quatro domínios: físico, psicológico, relações sociais e meio ambiente. 0 instrumento possui ainda duas questões que avaliam a qualidade de vida geral que, quando calculadas em conjunto, geram um escore independente dos domínios. A primeira refere-se à qualidade de vida de um modo geral e a segunda à satisfação com a própria saúde. As respostas dadas pelo sujeito devem referir-se somente às duas últimas semanas anteriores ao dia da coleta dos dados.

2 - IPAQ (International Physical Activity

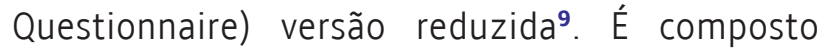
por oito questões, sendo um questionário sobre a duração e a intensidade da atividade física do indivíduo durante uma semana "habitual", realizada por pelo menos dez minutos contínuos, tanto em atividades ocupacionais quanto de locomoção, lazer ou prática esportiva.

3 - Questionário de classificação econômica da ABEP (Associação Brasileira de Empresas de Pesquisa). Tem por intuito estimar o poder de compra das pessoas e famílias a partir da divisão por classes econômicas. 0 instrumento é dividido em duas categorias, sendo que a primeira avalia a quantidade de posse de bens materiais e a segunda o nível de escolaridade do chefe da família. Cada item material e o nível de escolaridade possuem escores que são somados para estabelecer a classe econômica a que pertence o respondente, de acordo com escores próprios do instrumento ${ }^{10}$.

Todos os instrumentos foram aplicados em forma de entrevista, sendo que o entrevistador foi 
o responsável pelas anotações nos questionários correspondentes. O período de aplicação do instrumento ocorreu entre setembro e outubro de 2012, sendo que todos os participantes assinaram termo de consentimento livre e esclarecido e foi garantida a confidencialidade das informações e 0 direito a recusa em participar da pesquisa.

Os entrevistadores foram acadêmicos e docentes do curso de Educação Física do IFPR, previamente treinados para aplicação dos instrumentos utilizados. Os participantes assinaram o Termo de Consentimento Livre e Esclarecido de acordo com as normas da Resolução n 196/96.

As análises dos dados foram realizadas por meio da estatística descritiva de média e dispersão, sendo a significância atestada pelo teste $t$ de Student $(p<0,05)$ para amostras independentes, além da análise inferencial entre os domínios. Para análise estatística foi usado o programa SPSS versão $20.0{ }^{\circledR}$, sugerido por Fleck et $a^{8}{ }^{8}$.

0 projeto de pesquisa foi aprovado pelo Comitê de Pesquisa e Extensão do IFPR - Campus Palmas (COPE), de acordo com o Protocolo de Registro número 23408000578/2011-40 e Parecer 0058/2011.

\section{Resultados}

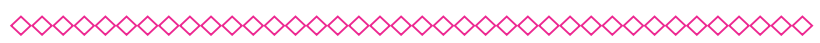

O objetivo da pesquisa foi analisar o nível de qualidade de vida dos servidores do IFPR Campus Palmas que se encontram na fase da senescência. No que concerne ao perfil sociodemográfico, 23 $(63,89 \%)$ são do sexo feminino e $13(36,11 \%)$ do masculino $(\mathrm{N}=36)$. A idade do grupo variou de 40 a 57 anos, com média de 46,36 anos.

Os indivíduos apresentaram a seguinte distribuição nas diversas classes que constituem a classificação econômica proposta pela ABEP (2010): Classe A2 (16,7\%), classe B1 (27,8\%), classe B2 $(27,8 \%)$, classe C1 $(11,1 \%)$ e classe C2 $(16,7 \%)$, conforme pode ser verificado no Gráfico 1.

No entanto, para efeitos de análise, os indivíduos foram divididos em dois grupos: 0 primeiro foi definido como "Renda Média/Alta" - RMA, sujeitos com níveis salariais acima de
R\$ $1.391,00$, e outro como "Renda Média/Baixa" - RMB, incluindo os demais sujeitos com faixa salarial abaixo desse valor. No grupo RMA estão inseridos $72,2 \%$ dos pesquisados, enquanto no RMB encontram-se $27,8 \%$ dos sujeitos.

No que se refere ao nível de atividade física (Gráfico 2), os indivíduos apresentaram distintas condições, considerando a classificação proposta no IPAQ: Muito ativos (13,9\%), ativos A $(27,8 \%)$, ativos $B(2,8 \%)$, insuficientemente ativos $(33,3 \%)$ e sedentários (22,2\%).

Para análise, os sujeitos foram divididos em dois grupos, sendo que no primeiro englobaram-se as categorias muito ativo, ativo $A$ e ativo $B$, sendo estes considerados como "ativos ou muito ativos" $(44,5 \%)$, enquanto na segunda foram inseridos os demais indivíduos, denominando o grupo como "insuficientemente ativos ou sedentários" (55,5\%).

A fim de verificar se os índices de QV dos pesquisados diferem em função do sexo, foram comparados os escores médios de cada domínio. Apesar de haver variação entre os escores encontrados para cada gênero, esta não foi significativa $(p<0,05)$. Analisando as médias dos domínios, é possível dizer que homens e mulheres estão satisfeitos quanto ao nível de QV, sendo o domínio das relações sociais considerado como o melhor índice $(M=4,30 \pm 0,56)$ e o ambiente sendo 0 $(M=3,78 \pm 0,70)$. No entanto, é importante destacar que todos os valores sugerem que o grupo pesquisado apresenta satisfação com seu nível de QV, conforme pode ser verificado na Tabela $\mathbf{1}$.

Para averiguar se a QV é influenciada nesse grupo pela condição econômica, foram comparados os índices médios obtidos pelos grupos. Foi encontrada uma diferença significativa entre RMA (Físico $=4,16 \pm 0,55$ e $p<0,002$; Psicológico $=4,18 \pm 0,49$ e $p<0,000$; Ambiente $=4,02 \pm 0,57$ e $\mathrm{p}<0,000$ ) e RMB (Físico $=3,51 \pm 0,46$ e $p<0,002$; Psicológico $=3,40 \pm 0,64$ e $p<0,000$; Ambiente $=$ $3,14 \pm 0,63$ e $p<0,000$ ).

No domínio das relações sociais, apesar de haver uma pequena variância (RMA $=4,33 \pm 0,61 ; R M B=$ $4,20 \pm 0,39)$, não houve diferença significativa entre os grupos $(p<0,527)$. No que se refere à condição econômica, percebe-se que o grupo com maior poder de renda apresentou melhores escores nos domínios físico, psicológico e do ambiente em 
relação aos que possuem menor poder aquisitivo. 0 domínio com os melhores escores médios foram as relações sociais $(M=4,30 \pm 0,56)$ e com menor valor aparece o domínio do ambiente $(M=3,78 \pm 0,70)$, semelhante aos dados da condição econômica.

Em relação ao nível de atividade física, percebese que não houve diferenças significativas entre os grupos em nenhum dos domínios, fato constatado na análise da Tabela 3. Isso sugere que o nível de atividade física para esse grupo não é ainda fator determinante para a QV. O quadro encontrado nas variantes anteriores se repetiu, sendo que 0 domínio com maior valor geral também foi o das relações sociais $(M=4,30 \pm 0,56)$ e o menor índice 0 do ambiente $(M=3,78 \pm 0,70)$ respectivamente.

\section{Discussão}

$\infty \times \infty \times \infty \times \infty \times \infty \times \infty \times \infty \times \infty \times \infty \times \infty \times \infty \times \infty \times \infty)$

O envelhecimento é uma das certezas da vida humana. Essa afirmação é explicada em estudos que destacaram o fato de o sujeito apresentar crescimento e desenvolvimento nos aspectos funcionais e biofisiológicos até aproximadamente os 30 anos de idade, período do ápice de tais funções. Em sequência, se inicia uma diminuição de tais capacidades, cuja velocidade de processamento pode ser minimizada de maneira relativa por estilos de vida saudáveis, 0 que inclui a prática regular de atividades físicas ou, contrariamente, ser acelerado por inações derivadas do comportamento sedentário ${ }^{2,11}$.

Nesse sentido, existem aspectos a serem analisados nos variados grupos, para entender a prevalência de determinado comportamento ou crenças acerca do próprio processo de envelhecimento, como retratamos com os sujeitos desta pesquisa.

No que se refere ao sexo, não parece haver diferenças significativas entre os grupos em relação aos índices de QV, como pôde ser verificado anteriormente na Tabela $\mathbf{1}$. 0 resultado contraria o encontrado por Alberte, Ruscalleda e Guariento ${ }^{12}$, que apresentou diferença significativa favorável às mulheres na percepção dos domínios físico, psicológico e ambiental, pressupondo, assim, que sujeitos do sexo feminino possuem uma visão mais positiva acerca da QV nas diferentes variáveis. Os autores atribuem essa diferença ao fato de que a maioria das mulheres ao envelhecer tende a morar com familiares, o que gera uma situação de maior segurança, convivência e suporte social, influenciando, assim, na forma como percebe sua vida e a qualidade desta.

Apesar desse estudo não evidenciar influência da idade nos escores de qualidade de vida, é importante considerar as diferenças de idade e sexo em estudos da QV. Quanto mais se avança na idade, piores são as concepções relacionadas a $\mathrm{QV}^{12,13}$. Em nosso estudo, um dos possíveis fatos que pode ter influenciado nos resultados é o perfil dos pesquisados, formado por docentes, técnicos administrativos e trabalhadores terceirizados de uma instituição federal, que, mesmo em processo de envelhecimento, parecem possuir condições mais positivas de desenvolver suas atividades laborais que outros segmentos da população.

Nas questões relacionadas à condição econômica, houve diferença significativa entre os grupos de renda média alta e renda média baixa nos domínios físico, psicológico e do ambiente (vide Tabela 2).

Em relação ao domínio físico, que abrange facetas relacionadas à dor, ao desconforto, à energia e à fadiga, ao sono e ao repouso, a diferença de resultado pode estar relacionada às características laborais das classes estratificadas, já que os indivíduos classificados como RMB são, em geral, trabalhadores terceirizados, que efetuam suas atividades em tarefas como carregar pesos, limpeza e conservação de ambientes internos (salas de aula, laboratórios etc.) e externos (jardins, calçadas etc.), onde se exige força física e há uma maior possibilidade de adquirir lesões derivadas do esforço se comparadas às atividades desenvolvidas por docentes e técnicos administrativos. É importante destacar que indivíduos com mais de 60 anos sentem mais os efeitos dessas atividades que indivíduos com idade inferior ${ }^{7}$. Como o grupo em questão está em um ponto intermediário entre a idade adulta e a velhice, as dores relacionadas ao modo de vida anterior, podem ser responsáveis pela diferença positiva obtida nesse domínio em favor do grupo RMA, cujas atividades demandam menor esforço físico. 
No domínio psicológico, em que estão inseridas as facetas com sentimentos positivos e negativos, autoestima, cognição e satisfação com a imagem corporal, os resultados demonstraram diferença significante no aspecto econômico, onde o grupo RMA apresentou índices melhores que $\mathrm{RMB}$ em relação à $\mathrm{QV}$. $\mathrm{O}$ poder de renda tem sido relatado como fator determinante para que o indivíduo considere possuir uma boa QV. Autores defenderam em seus estudos que as doenças psicológicas estão associadas ao prejuízo na QV dos sujeitos, sugerindo, ainda, que aqueles acometidos por estas apresentam QV menor do que a população em geral ${ }^{12}$. Os quadros de alterações psicológicas ocasionam inserção social limitada em variadas áreas como relações interpessoais, lazer, trabalho e estado geral de saúde. No caso do grupo em questão, os sujeitos com RMA apresentam melhores condições de tempo para conviver com as famílias e acesso a oportunidades de lazer, já que sua carga de trabalho é de 40 horas semanais, o que pode refletir no resultado, enquanto os de RMB em geral efetuam trabalhos extras para complementar a renda, não possuindo assim tempo para convivência familiar ou lazer. Da mesma maneira, acredita-se que, devido ao grupo RMA possuir mais anos de estudos que 0 grupo RMB, tal condição facilite a dinamicidade das relações, a maior facilidade de comunicação e interação social, apresentando, assim, uma concepção melhor de QV.

O domínio das relações sociais, que possui facetas que avaliam as relações pessoais, 0 suporte social e a atividade sexual, foi o que apresentou melhor escore médio e não indicou diferença significativa entre os grupos, fato encontrado também em estudos com populações semelhantes ${ }^{12,14}$. O resultado sugere que os sujeitos pesquisados entendem não haverem maiores dificuldades para inserção no meio comunitário ou familiar. Da mesma maneira, parecem demonstrar que estes sentem-se seguros nas suas relações com outras pessoas (familiares ou sociais). Como o grupo em estudo convive diariamente e os espaços do local de trabalho admitem um acesso razoável entre todos, acredita-se ser esse um dos fatores que permitiu serem as relações sociais o domínio com maior escore.
O domínio do ambiente teve diferença significativa entre os grupos e destaca-se por apresentar os menores índices entre todos os domínios. Sugere-se que os sujeitos da pesquisa entendem que os espaços onde convivem não sejam adequados. Uma pesquisa encontrou resultados semelhantes em idosos, em que 0 domínio ambiente apresentou os escores mais baixos $^{15}$. Esse estudo indicou, ainda, que a visão do ambiente pode estar relacionada às inseguranças e incertezas da sociedade brasileira contemporânea, fato considerado como coerente também para os resultados aqui encontrados. Contrariamente a isso, um estudo com gestores de empresas no Rio Grande do Sul relatou ser o ambiente o domínio com melhor índice ${ }^{16}$. Observando os participantes dessa pesquisa, sugere-se que os pesquisados estão sentindo dificuldades de adaptação ao meio, seja ele familiar ou social, além de possuírem poucos espaços ou tempo para o lazer, fator que se reflete no baixo conceito do domínio em relação aos demais.

No que se refere ao nível de atividade física, não houve relação significante entre os grupos, 0 que sugere que para esses indivíduos essa variável ainda não trouxe grandes impactos. 0 resultado contraria a maioria dos estudos, que apresenta relação positiva entre a atividade física e a QV. A atividade física foi apontada, ainda, como benéfica nas diferentes faixas etárias para aumento da força muscular, autoestima, autoimagem, percepção corporal, entre outros ${ }^{17-19}$.

É importante ressaltar que apesar da maioria dos estudos concordarem que a atividade física é determinante na melhoria da concepção de QV dos indivíduos, ela apresenta efeito transitório na vida das pessoas, já que menores escores da prática de AF resultam na diminuição dos índices de qualidade de vida 20 .

0 fato de as atividades físicas apresentarem um determinado tempo para terem sua eficácia percebida parece estar relacionado à falta de estímulo dos pesquisados no que se refere à sua prática. Isso é constatado no fato de haver na instituição de onde são oriundos um programa de ginástica laboral em que há um percentual de cerca de $40 \%$ de indivíduos que optaram pela não participação, o que provavelmente reflete 
na condição apresentada neste trabalho. Aqui se encontra uma lacuna investigativa, onde é necessário questionar por que a atividade física não se torna habitual, já que está comprovado que sua prática eleva a percepção de elementos que compõem os quadros de QV.

\section{Conclusão}

$\infty \times \infty \times \infty \times \infty \times \infty \times \infty \times \infty \times \infty \times \infty \times \infty \times \infty \times \infty \times \infty)$

A qualidade de vida é base de várias condições potenciais que podem afetar a forma como 0 indivíduo percebe o mundo, os comportamentos cotidianos e os sentimentos advindos de suas relações, o que inclui, porém não limita, a condição de saúde. Se partirmos dessa premissa, a QV colocase como impactante no estado geral de saúde e na capacidade de viver plenamente.

Os dados encontrados não apresentaram relações significativas entre a QV e o nível de atividade física. No entanto, o nível de condição econômica parece ser fundamental na concepção da QV dos sujeitos, apresentando índices melhores em indivíduos com maior poder de renda.

Os dados encontrados refletem-se em outros estudos com populações variadas, conforme foi verificado no decorrer deste trabalho, o que sugere que o grupo analisado está dentro dos padrões de normalidade para um público em processo de envelhecimento. Porém, a velhice não parece ser ainda um fator que interfere nas percepções de QV dos sujeitos da pesquisa.

Apesar de o nível de atividade física não ter apresentado significância, os diversos estudos apresentados na discussão demonstram que pessoas em processo de envelhecimento devem usar desta para ampliar suas condições de permanecer ativas e autônomas por um maior período de tempo.

A limitação existente neste estudo, comum às pesquisas que utilizam o corte transversal, é o fato de não permitirem estabelecer relação de causa e efeito, ou seja, não explicam a origem dos dados encontrados. Por sua característica quantitativa, não é possível estabelecer a percepção dos indivíduos acerca do tema da pesquisa e suas variáveis, pois não se buscam as fontes indicadoras das respostas, somente possível se houvesse um acompanhamento temporal.

\section{Agradecimento}

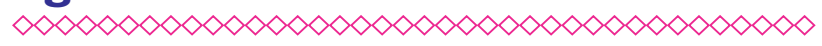

Aos servidores participantes que foram essenciais para realização do estudo.

\section{Referências}

$\infty \times \infty \times \infty \times \infty \times \infty \times \infty \times \infty \times \infty \times \infty \times \infty \times \infty \times \infty \times \infty$

1. Instituto Brasileiro de Geografia e Estatística. Coordenação de População e Indicadores Sociais. Estudos e Pesquisas. Informação Demográfica e Socioeconômica. Síntese de Indicadores Sociais. Rio de Janeiro: IBGE, 2010.

2. Veras R. Envelhecimento populacional contemporâneo: demandas, desafios e inovações. Rev. Saude Públ. 2009; 43(3): 548-54.

3. Jobim FARC, Jobim EFC. Atividade física, nutrição e estilo de vida no envelhecimento. UNOPAR Cient. Ciênc. Biol. Saúde. 2015; 17(4):298-308.

4. World Health Organization. Development of the World Health Organization. WHOQOL-BREF quality of life assessment: the WHOQOL Group. Psychol Med 1998; 28:551-58.

5. Minayo MCS, Hartz ZMA, Buss PM. Qualidade de vida e saúde: um debate necessário. Cienc. Saúde Colet. 2000; 5(1): 7-18.

6. Ribeiro LA, Santana LC. Qualidade de vida no trabalho: fator decisivo para o sucesso organizacional. RIC Cairu. 2015; 2(2): 75-96.

7. Ribeiro CAO, Campos LNM. Qualidade de vida no trabalho. Tecer. 2012; 2(2):28-39.

8. Fleck MPA, Louzada S, Xavier M, Chachamovich E, Vieira $G$, Santos L, et al. Aplicação da versão em português do Instrumento de avaliação de qualidade de vida da OMS (WHOQOL-100). Rev. Saúde Públ. 1999b; 33(2):198-205.

9. Pardini R, Matsudo S, Araújo T, Matsudo V, Andrade E, Braggion G, et al. Validação do 
questionário internacional de nível de atividade física (IPAQ-versão 6): estudo piloto em adultos jovens brasileiros. Rev. Bras. Cienc. Mov. Brasília. 2001; 9(3): 45-51.

10. Associação Brasileira de Empresas de Pesquisa (ABEP). Critérios de Classificação Econômica Brasil. 2015. Disponível em: http:// www.abep.org/ criterio-brasil.

11. Krug RR, Lopes MA, Mazo GZ. Barreiras e facilitadores para a prática da atividade física de longevas inativas fisicamente. Rev. bras. ciênc. esporte. 2015; 2(1): 57-64.

12. Alberte JSP, Ruscalleda RMI, Guariento ME. Qualidade de vida e variáveis associadas ao envelhecimento patológico. Rev. Soc. Bras. Clin. Med. 2015; 13(1): 32-9.

13. Lima DL, Lima MAVD, Ribeiro CG. Envelhecimento e qualidade de vida de idosos institucionalizados. RBCEH. 2012; 7(3): 346-356.

14. Pucci GCMF, Rech CR, Fermino RC, Reis RS. Associação entre atividade física e qualidade de vida em adultos. Rev. Saúde Públ. 2012; 46(1): 166-79.

15. Tavares DMS, Dias FA. Capacidade funcional, morbidades e qualidade de vida de idosos. Texto Contexto - Enferm. 2012; 21(1): 112-20.

16. D'Amico SM, Monteiro JK. Características de personalidade e qualidade de vida de gestores no Rio Grande do Sul. Rev. Adm. Contemp. 2012; 16(3): 381-96.

17. Pelozatto D, Fernandes RA. Relação entre qualidade de vida e atividade física: uma revisão sistemática da literatura nacional. Colloq. Vitae. 2011; 3(2): 54-58.

18. Orlando MM, Silva MSP, Lombardi Junior I. A influência da prática de atividade física na qualidade de vida, força muscular, equilíbrio e capacidade física de idosos. Rev. bras. geriatr. gerontol. 2013; 16(1): 117-126.

19. Scheffer MLC, Pilatti LA, Kovaleski JL. Qualidade de vida e atividade física na literatura. Espacios. 2015; 36(3): 7-12.
20. Silva MF, Goulart NBA, Lanferdini FJ, Marcon $M$, Dias P. Relação entre os níveis de atividade física e qualidade de vida de idosos sedentários e fisicamente ativos. Rev. bras. geriatr. gerontol. 2012;15(4):635-642. 


\section{Anexos}

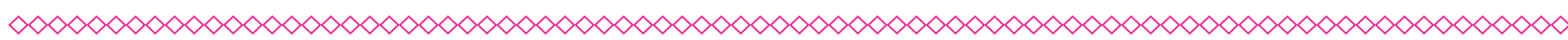

Gráfico 1 - Distribuição percentual da condição econômica dos servidores do IFPR Campus Palmas

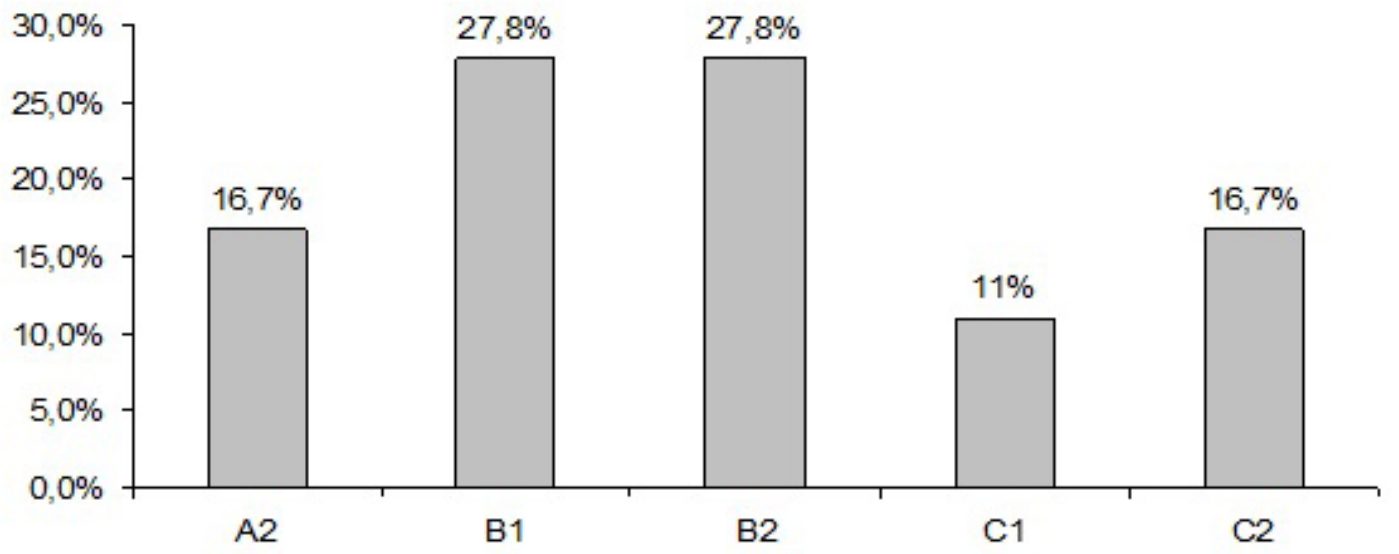

(clique para voltar ao texto)

Gráfico 2 - Distribuição percentual do nível de atividade física dos servidores do IFPR Campus Palmas

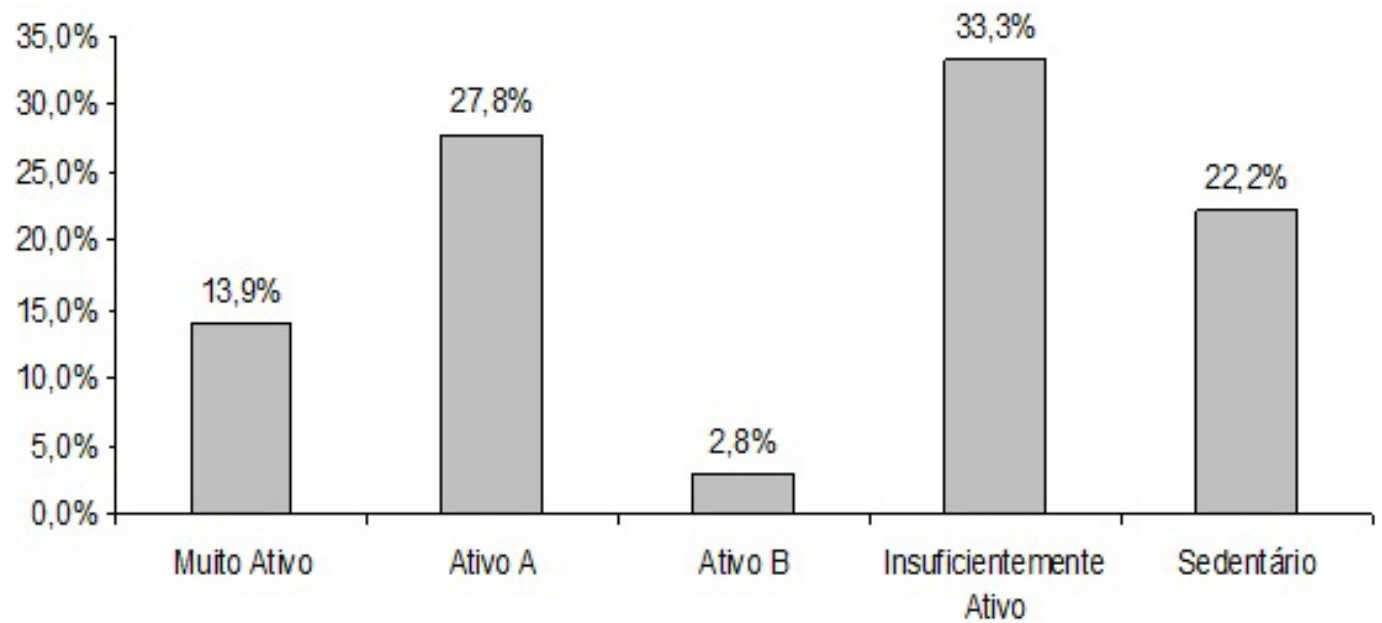

(clique para voltar ao texto) 
Tabela 1 - Comparação dos servidores do IFPR Campus Palmas acerca da percepção de qualidade de vida em função do sexo

\begin{tabular}{lllll}
\hline & & N & Média/dp & p \\
& Masculino & 13 & $4,14 \pm 0,67$ & \\
Domínio Físico & Feminino & 23 & $3,89 \pm 0,55$ &, 224 \\
& Total & 38 & $3,98 \pm 0,60$ & \\
Domínio Psicológico & Masculino & 13 & $4,15 \pm 0,60$ & \\
& Feminino & 23 & $3,86 \pm 0,64$ & .178 \\
& Total & 36 & $3,96 \pm 0,63$ & \\
Domínio das & Masculino & 13 & $4,26 \pm 0,60$ & \\
Relaçées Sociais & Feminino & 23 & $4,32 \pm 0,55$ & .752 \\
& Total & 36 & $4,30 \pm 0,56$ & \\
Domínio do Ambiente & Masculino & 13 & $3,89 \pm 0,53$ & \\
& Feminino & 23 & $3,71 \pm 0,79$ & .462 \\
& Total & 38 & $3,78 \pm 0,70$ & \\
\hline
\end{tabular}

(clique para voltar ao texto)

Tabela 2 - Comparação da percepção de qualidade de vida em função da condição econômica dos servidores do IFPR Campus Palmas

\begin{tabular}{|c|c|c|c|c|}
\hline & & $\mathrm{N}$ & Média/dp & $\mathbf{p}$ \\
\hline \multirow{4}{*}{ Domínio Físico } & Renda Média & 28 & $4,16 \pm 0,55$ & \\
\hline & Renda Baixa & 10 & $3,51 \pm 0,46$ & .002 \\
\hline & Total & 36 & $3,98 \pm 0,60$ & \\
\hline & Renda Média & 28 & $4,18 \pm 0,49$ & \\
\hline \multirow[t]{2}{*}{ Domínio Psicológico } & Renda Baixa & 10 & $3,40 \pm 0,64$ & .000 \\
\hline & $\begin{array}{l}\text { Total } \\
\text { Renda Média }\end{array}$ & $\begin{array}{l}36 \\
26\end{array}$ & $\begin{array}{l}3,96 \pm 0,63 \\
4,33 \pm 0,61\end{array}$ & \\
\hline \multirow[t]{2}{*}{ Domínio das Relações Sociais } & Renda Baixa & 10 & $4,20 \pm 0,39$ & .527 \\
\hline & $\begin{array}{l}\text { Total } \\
\text { Renda Média }\end{array}$ & $\begin{array}{l}36 \\
28\end{array}$ & $\begin{array}{l}4,30 \pm 0,56 \\
4,02 \pm 0,57\end{array}$ & \\
\hline \multirow[t]{2}{*}{ Domínio do Ambiente } & Renda Baixa & 10 & $3,14 \pm 0,63$ & .000 \\
\hline & Total & 36 & $3,78 \pm 0,70$ & \\
\hline
\end{tabular}


Tabela 3 - Comparação da percepção de qualidade de vida em função do nível de atividade física pelos servidores do IFPR Campus Palmas

\begin{tabular}{|c|c|c|c|c|}
\hline & & $\mathrm{N}$ & Média / dp & $p$ \\
\hline & Ativo ou muito ativo & 16 & $4,09 \pm 0,60$ & \\
\hline \multirow[t]{3}{*}{ Domínio Físico } & Pouco Ativo ou Sedentário & 20 & $3,89 \pm 0,60$ &, 334 \\
\hline & Total & 36 & $3,98 \pm 0,60$ & \\
\hline & Ativo ou muito ativo & 16 & $4,03 \pm 0,70$ & \\
\hline \multirow[t]{2}{*}{ Domínio Psicológico } & Pouco Ativo ou Sedentário & 20 & $3,91 \pm 0,59$ &, 571 \\
\hline & Total & 36 & $3,96 \pm 0,63$ & \\
\hline \multirow{3}{*}{ Domínio das Relações Sociais } & Ativo ou muito ativo & 16 & $4,35 \pm 0,52$ & \\
\hline & Pouco Ativo ou Sedentário & 20 & $4,25 \pm 0,59$ &, 584 \\
\hline & Total & 36 & $4,30 \pm 0,56$ & \\
\hline \multirow{3}{*}{ Domínio do Ambiente } & Ativo ou muito ativo & 16 & $3,91 \pm 0,71$ & \\
\hline & Pouco Ativo ou Sedentário & 20 & $3,68 \pm 0,70$ &, 333 \\
\hline & Total & 36 & $3,78 \pm 0,70$ & \\
\hline
\end{tabular}

